\title{
THE RELATION BETWEEN CHROMOSPHERIC AND PHOTOSPHERIC STRUCTURES IN SUNSPOT GROUPS
}

\author{
V. BUMBA and P. AMBROŽ \\ Astronomical Institute of the Czechoslovak Academy of Sciences, Ondřejov Observatory, Czechoslovakia
}

\begin{abstract}
Using the high resolution photographs of the photosphere taken at the Ondřejov observatory a comparison of positions and forms of some chromospheric features with the distribution of photospheric fine structure morphological elements in the August 1972 active region has been made. The close relationship between active filaments, chromospheric threads, fibrils and photospheric small nuclei, dark interpenumbral and intergranular spaces or rudimentar penumbra is shown. Some considerations are demonstrated with the aid of computed models of the horizontal component of the magnetic vector simulating the real distribution of magnetic fields in the studied region.
\end{abstract}

\section{Introduction}

Recently, a relatively large number of papers studying the individual characteristic elements of chromospheric fine structure and their relation to the photospheric magnetic fields has been published. This work has been done mostly by the Big Bear Solar Observatory group (Zirin, 1970; Prata, 1971; Foukal, 1971a, b) as well as by others. In this paper, we study the relationship of the described chromospheric features to features seen in high resolution photgraphs of the photosphere, especially to those of sunspot structures.

For example, solar surges have been demonstrated to arise from certain regions of sunspots (Roy, 1973; Gopasyuk et al., 1963; Gopasyuk and Obir, 1963). Although these regions have been characterized magnetically their morphology in the photosphere has not yet been examined. Also Frazier (1972) has shown that the ends of arch filaments are rooted in groups of 'knots' of the photospheric magnetic field.

These facts led us to an investigation of the relations of filaments, chromospheric threads, arches and fibrils to the underlying morphological details of sunspots, their penumbras and surrounding photosphere. An initial comparison of relatively low resolution chromospheric $\mathrm{H} \alpha$ photographs $\left(\sim 2^{\prime \prime}\right)$ demonstrated that a great number of chromospheric features in an active region are related to certain peculiarities in spot penumbras or to small nuclei around large spots.

In the present note, we extend this study using good observations of the large August 1972 active region.

\section{Observational Material}

The great August 1972 sunspot group has been observed photographically at our observatory starting from July 31 till August 10 . During this time interval a large number of photospheric photographs were obtained. The instruments and method of observation were described by Bumba et al. (1973). Each day many series of photo- 
graphs with different exposures, usually several tens of minutes apart have been made. The quality of photographs varied from day to day as well as during a given day depending on weather and seeing conditions. But in some exposure series photographs with resolution around 1 " or better may be found.

Good quality photographs of the chromosphere from the Big Bear Solar Observatory Flare Film 1972 (taken for the major part in the hydrogen $\mathrm{H} \alpha$ line) have been used.

\section{Results}

In the photosphere, the sunspot group was characterized morphologically by several large umbras (their development and magnetic field polarity distribution may be seen, for example in Bumba, 1973), and by an extensive penumbra, the greatest part of which was irregular. The penumbra enclosed many small umbral nuclei or darker, elongated spaces between the bright penumbral fibrils. Also, the photosphere penetrates into this penumbra in the form of gulfs, unusually bright filaments, chains of granules, light bridges etc. Only a small part of the penumbra was more or less regular, with radial fibrils and relatively sharp boundary with the photosphere. Among the more interesting features of the group were the light bridges. These had several different forms, some of them developing so vigorously that they appeared to divide the largest umbra and push its pieces apart. Some of these light bridges were in the form of a penumbra with very elongated, slightly curved fibrils coinciding with the magnetic field polarity boundary, which is very unusual. The special appearance of penumbral structures can be used by a skilled observer as an indicator of the presence of opposite polarity fields and as tracers of the magnetic field direction in the way that fibrils and filaments are used in the chromosphere. In the photosphere, of course, these associations may be checked by direct measurements of the magnetic field strength and polarity.

The general structural characteristics of the chromosphere above the active region were: a system of active region filaments (indicating the main polarity boundary) composed from individual fibrils connecting opposite polarities; regions formed by long fibrils or threads stretched out more or less radially from the group (magnetically characterized by presence of both polarities in islands); and regions without fibrils or threads. In the latter regions the dark space above the umbra and penumbra merges continuously into the quiet chromosphere far away from the spot. The quiet chromospheric structure around the spot group is characterized magnetically by a supergranular cell of predominantly one polarity which is the same as the polarity of the leader spot. There are also bright features and several chromospheric flares developed in the region.

Comparing the chromospheric and phtospheric structures, one sees that the chromospheric regions with long fibrils and threads are connected with the regions of disturbed penumbra, i.e., with those regions where changes in the distribution and form of penumbral structures and umbral nuclei take place (see Figures 1 and 2). On the other hand the chromospheric regions without distinct structures and with direct transition 

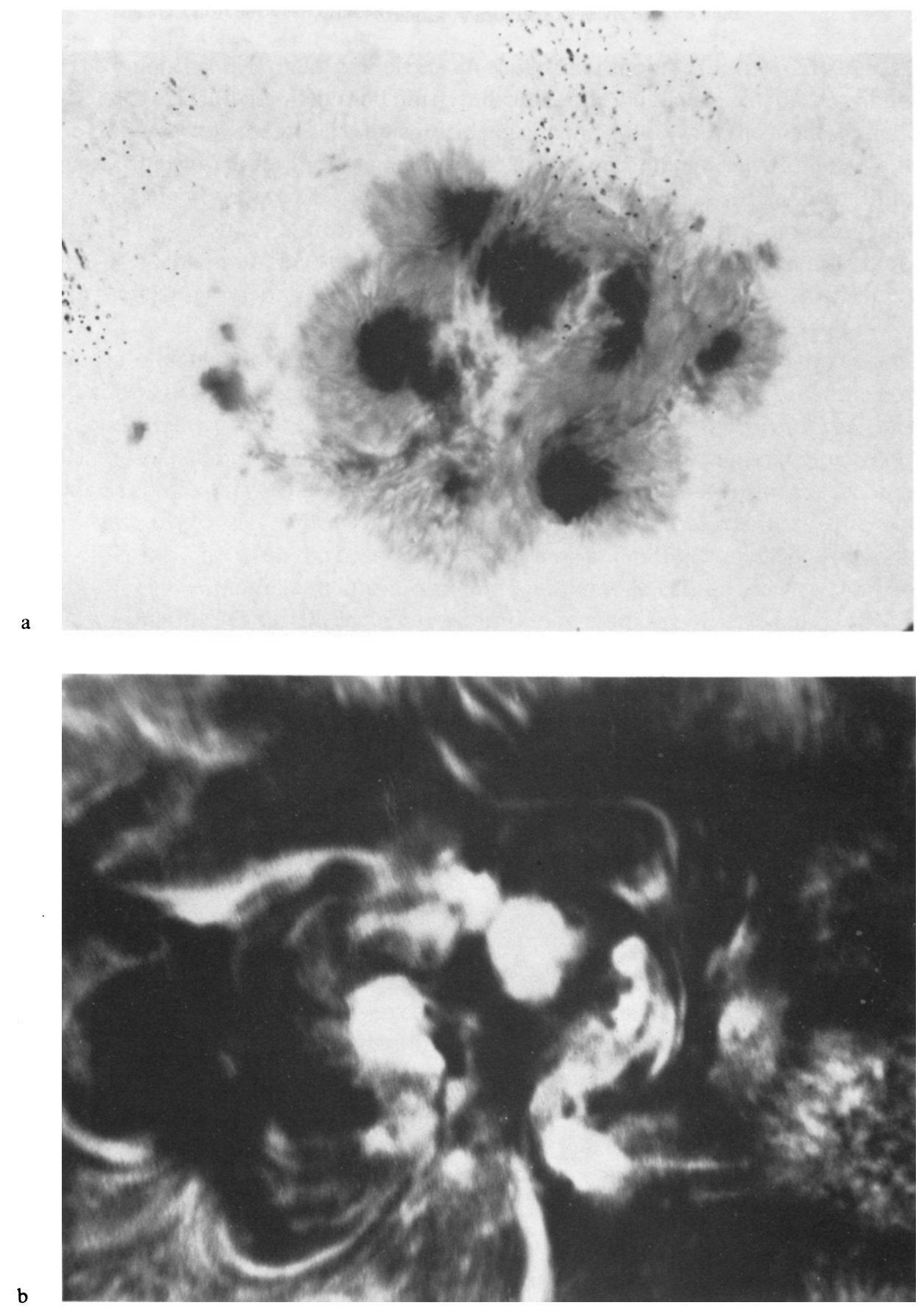

Figs. 1a-b. Photograph of the photosphere in the August 1972 active region taken in Ondřejov on August 4, at $14^{\mathrm{h}} 31^{\mathrm{m}} 34^{\mathrm{s}}$ UT compared with the negative photograph of the same active region taken from the Big Bear Solar Observatory Flare Film in $\mathrm{H} \alpha$ at $19^{\text {h }} 30^{\mathrm{m}} 50^{\mathrm{s}}$ UT. Both photographs have the same scale and orientation. Using a sheet of transparent paper one can compare the mutual positions of details in the photosphere and chromosphere.

A chromospheric region with long fibrils and threads related to a photospheric region of disturbed penumbra may be seen, for example, in the lower parts of both photographs. Also, a region without distinct structures in the chromosphere and with a normal quiet penumbra is well visible on the right sides of both pictures. 


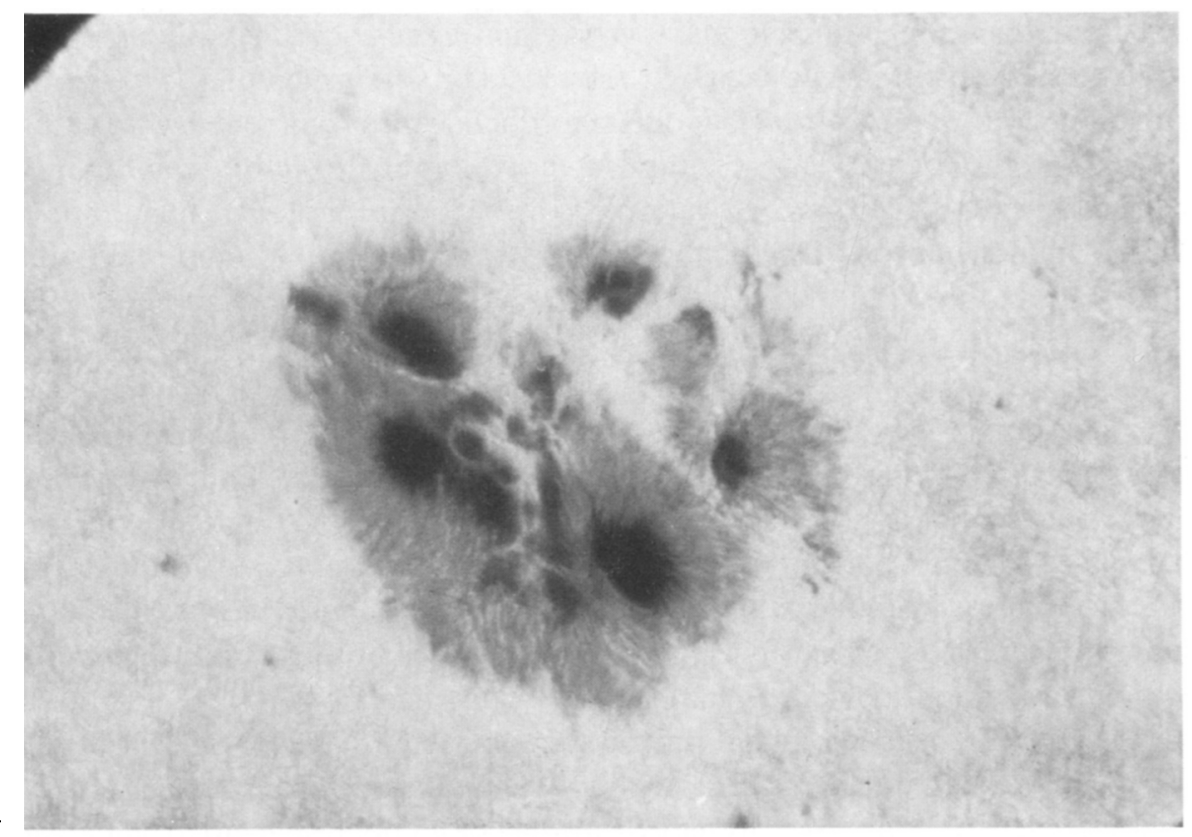

\section{a}

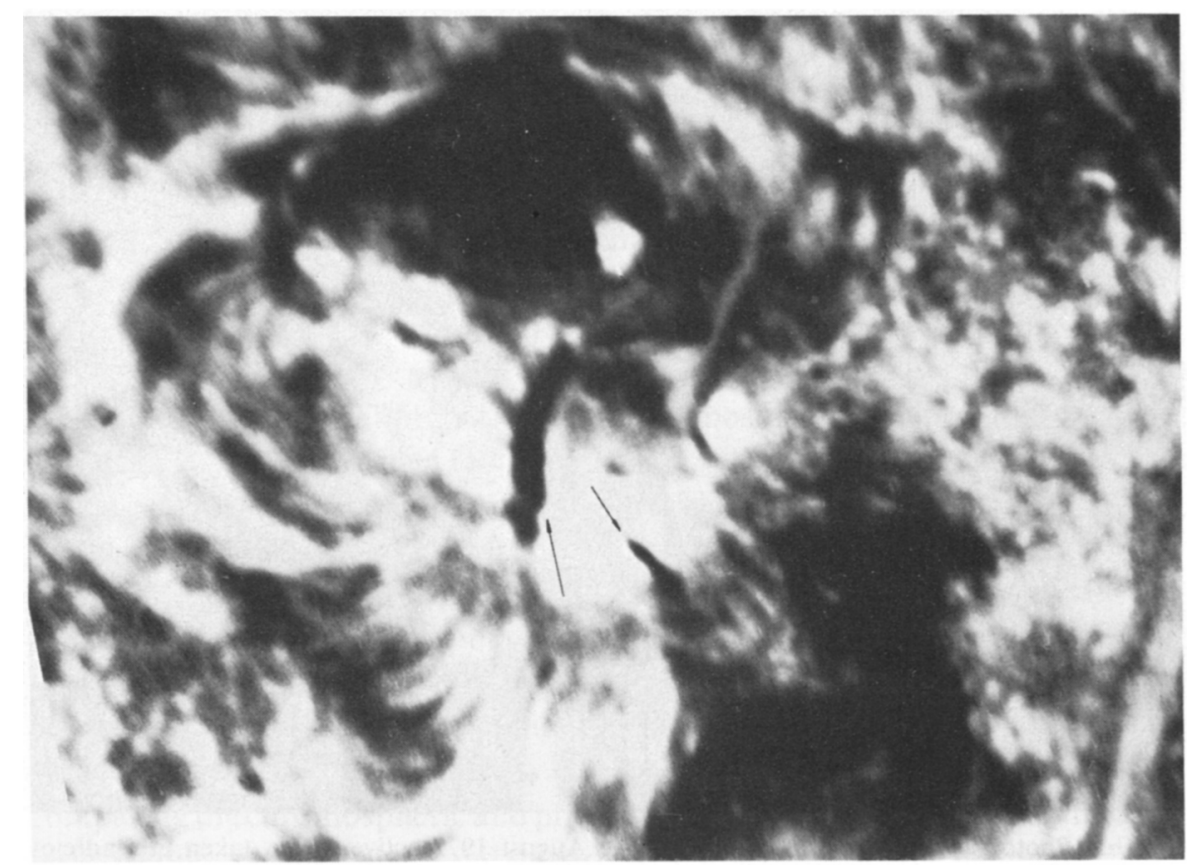

Figs. 2a-b. Combination of the same photospheric photograph $\left(15^{\text {h }} 09^{\mathrm{m}} 36^{\mathrm{s}} \mathrm{UT}\right)$ and a chromospheric picture taken during the very first stage of large flare development $\left(14^{\mathrm{h}} 59^{\mathrm{m}} 10^{\mathrm{s}}\right.$ UT) 1972 , August 7 . The brightening of emission above light bridges may be seen. 
into the quiet chromosphere coincide with the regular umbras (see also Figures 1 and 2). The active region filaments and fibrils indicating the magnetic field line distribution in the active region and its vicinity coincide inside the group with the highest gradient of magnetic field across the boundary of polarities and therefore with the region of elongated, slightly curved bright fibrils of penumbra forming a special form of light bridges in this group (Figures 1 and 2). The individual fibrils of this filament join the groups of small umbral nuclei of opposite polarity placed close to the polarity boundary. In these latter regions there are fast changes due to growth as well as to disintegration.

Exact comparison of the positions of ends of individual fibrils, threads and filament fibrils shows that, for the most part, those in the nearest neighbourhood of the group are closely related to the darker elongated spaces between the penumbral bright fibrils, to small spots and nuclei without penumbra, to darker enlarged intergranular space or rudimentary penumbra in the vicinity of the great spots or to the secondary small spot groups developed around the main group (Figures a and 2). Due to the time differences between the best observing periods at Ondřejov and Big Bear, only a few of the photospheric and chromospheric photographs can be directly compared. (Pictures obtained at Ondřejov in late afternoon overlap the observing period at Big Bear.) By studying the development of those photospheric details in which the chromospheric features seem to be rooted during the whole days it is possible to say that, the fibrils are rooted in regions where the small spots or darker features are changing only slowly in position (Figure 2).

Chromospheric emission is usually very closely associated with underlying light bridges (Figures 1 and 2). During the first phase of flare occurrence the brightened regions of the chromosphere coincide with the light bridges even better (Figure 2). The flare brightening develop along the length of the light bridges and was still visible in these locations during the declining phase of the flare. The development of flare emission on these high resolution $\mathrm{H} \alpha$ photographs resembles the situation observed in the calcium chromosphere (Bumba and Howard, 1965).

Only few examples may be given concerning the dynamics of related chromospheric and chromospheric features. Westwards from the main group, a small regular spot of negative polarity and having, for a short time, a rudimentary penumbra was observed. This spot acted during the first few days as an attractive center for a system of fibrils and threads spiraling around it and with matter flowing into the spot. After August 5 the existence of this spot had no further influence on the distribution of chromospheric structures around and above the spot, although its photospheric appearance did not seem to change.

\section{Discussion}

What conclusion can be made from the fact that the greatest part of chromospheric features above an active region is rooted in small photospheric dark features on the periphery of a sunspot group or around it? The intensity of the magnetic field in such photospheric objects is at least of the order of $1000 \mathrm{G}$ (Bumba, 1967). These objects 


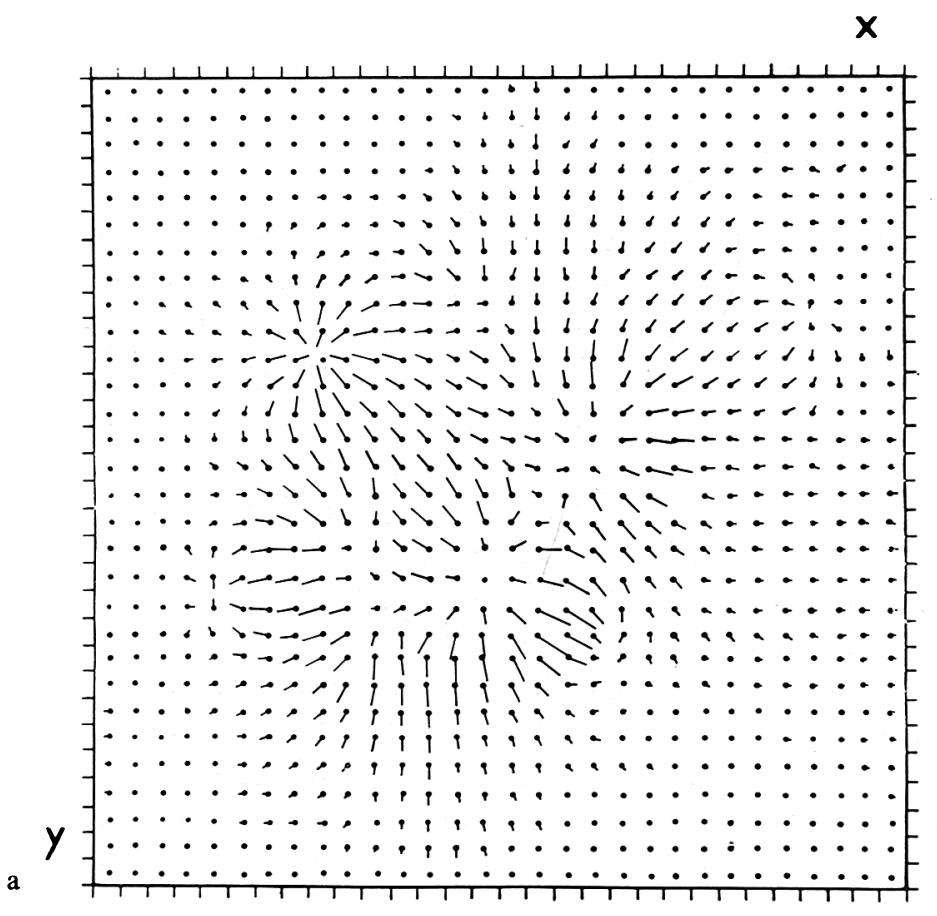

b

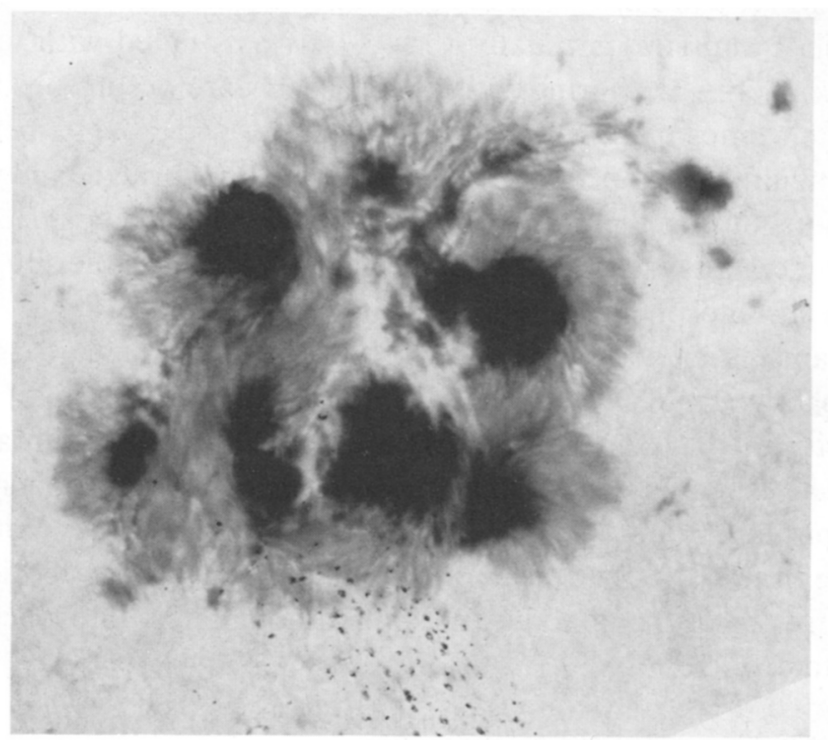

Figs. 3a-b. 1972, August 4: Combination of the photospheric photograph and the directions of the horizontal component of the magnetic vector slightly (the distance of two points in one line on the graph) above the studied group, calculated in potential approximation with the aid of a matrix corresponding to the photographically obtained magnetic field intensity and polarity distribution. The scale and orientation of both figures is the same. 

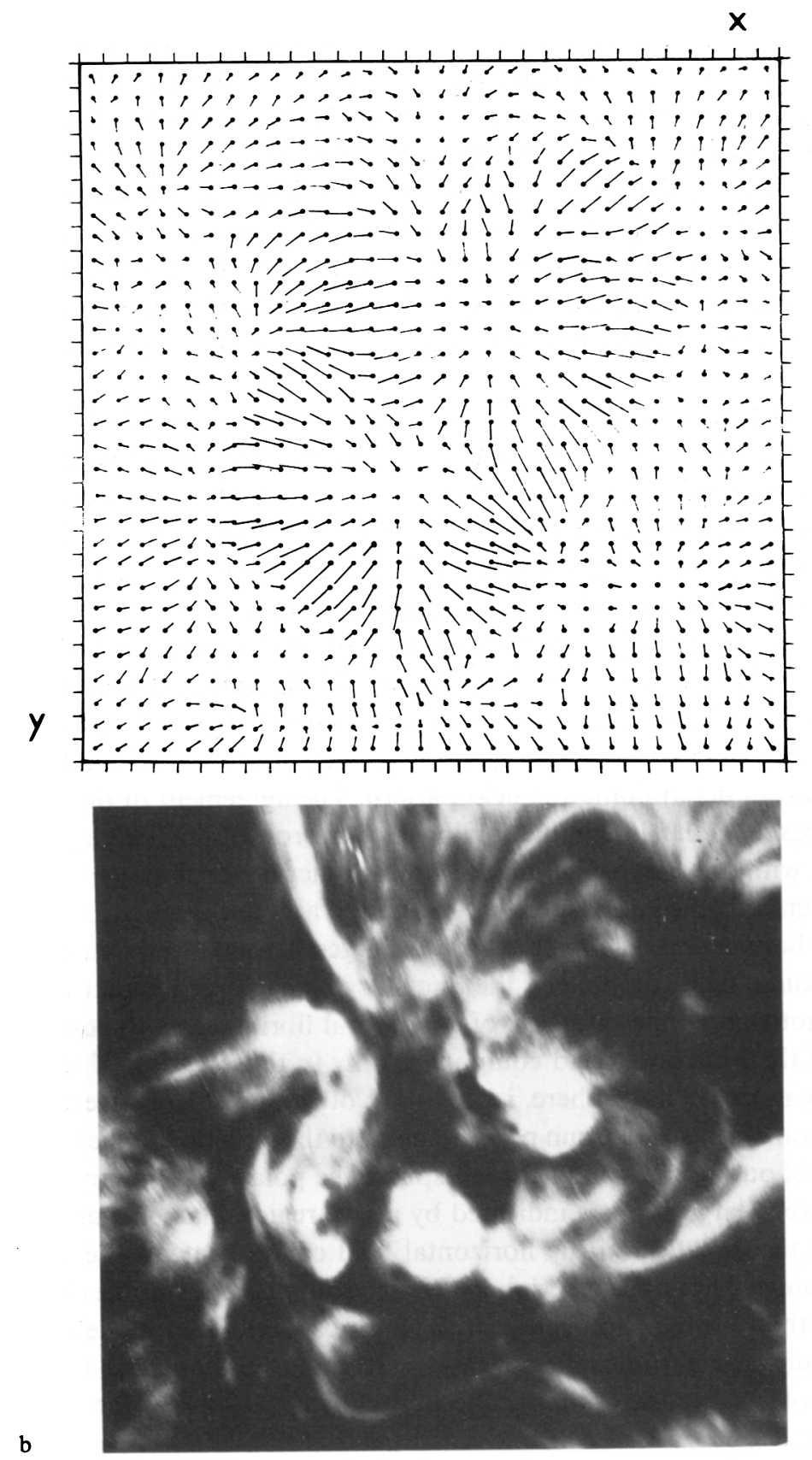

Figs. 4a-b. 1972, August 4: Comparison of the chromospheric photograph with the graph of distribution of directions of the horizontal component of the magnetic vector slightly (the distance of two points in one line of the graph) above the studied group, calculated in potential approximation with the aid of a matrix in which the magnetic field is represented by magnetic points with the same intensity, polarity distribution corresponding to that of a magnetogram (Livingston, 1973). The scale and orientation of both pictures is the same. 
are relatively unstable. Their life-time seems to be of the order of hours, although the region in which they occur lasts for days. Usually one takes these features as of 'secondary' importance in an active region. As has been already demonstrated (Foukal, 1971a, b) individual fibrils join the opposite polarities of magnetic fields often lying along the polarity boundary and they lie very low. Thus, the structure as we envision it seems to be far from hitherto existing theoretical models. At the same time another question seems to arise: why does the morphology of the chromosphere above an active region seem to be influenced only by these 'secondary' eelements of photospheric structure and so little affected by the huge umbras with very high values of magnetic flux?

To study at least some aspects of this question we have calculated by a potential approximation (Schmidt, 1963) two models of the horizontal component of the magnetic vector slightly above the photospheric level. We have simulated the real distribution of solar magnetic fields for 1972 August 4, in two ways: (a) by a matrix in which the magnetic field is represented by magnetic points with the same intensity and polarity distribution as that of a magnetogram (Livingston, 1973);(b) by a matrix corresponding to the total magnetic field intensity and polarity distribution obtained photographically (Roma Photographic Journal of the Sun). Comparing the resulted field with photospheric and chromospheric structure (Figures 3 and 4), we see that in such a static model the magnetic fields of all magnetic features (small as well as big) are important in determining the field pattern. The agreement of the magnetic map (Figure 4), based on the fine simulation in which the role of smaller magnetic elements is included, with the photospheric and chromospheric morphology is better than in the case when only the larger spots are taken into account (Figure 3). (In our models the polarity boundary is demonstrated by the largest arrows representing the direction of the horizontal field component, the boundary being perpendicular to them.)

In the photosphere the direction of penumbral fibrils seems to coincide with the direction of the horizontal field component only in those region of spot penumbra bordered by normal photosphere, i.e., without other spots. In between larger spots, the penumbral fibrils seem to run perpendicular to the direction of the horizontal field component of our model. In the chromosphere the polarity boundary in closed field regions between large spots is indicated by active region filaments crossing perpendicularly to the direction of the horizontal field component. In the case when the polarity boundary lies on the periphery of the group, having on one side large spots and on the other side the photosphere with chromospheric network fields and secondary spots only, the chromospheric features seem to lie along the horizontal field component of our model, outlining the well known patterns of the chromospheric morphology.

\section{References}

Bumba, V.: 1967, Solar Phys. 1, 371.

Bumba, V.: 1973, World Data Center A for Solar-Terrestrial Physics, Report UAG-28, Part I, July 1973, p. 82.

Bumba, V. and Howard, R.: 1965, Astrophys. J. 142, 796. 
Bumba, V., Ranzinger, P., and Suda, J.: 1973, Bull. Astron. Inst. Czech. 24, 22.

Foukal, P.: 1971a, Solar Phys. 20, 298.

Foukal, P.: 1971b, Solar Phys. 19, 59.

Frazier, E. N.: 1972, Solar Phys. 26, 130.

Gopasyuk, S. I. and Ogir, M. B.: 1963, Izv. Krymsk. Astrofiz. Obs. 30, 185.

Gopasyuk, S. I., Ogir, M. B., and Tsap, T. T.: 1963, Izv. Krymsk. Astrofiz. Obs. 30, 148.

Livingston, W. C.: 1973, World Data Center A for Solar-Terrestrial Physics, Report UAG-28, Part I, July 1973, p. 95.

Prata, S. W.: 1971, Solar Phys. 20, 310.

Roy, J.-R.: 1973, Solar Phys. 28, 95.

Schmidt, H. U.: 1963, AAS-NASA Symp. on the Physics of Solar Flares, p. 107.

Zirin, H.: 1970, Solar Phys. 14, 328.

\section{DISCUSSION}

Zirin: I agree with you that there is a strong sheer in the magnetic field that is diverging and going to far away places, some very near the surface. This was our interpretation in the case of these big flares which is also very well seen in the case of the August 1966 flares. One sees that nearly all of the flux in these newly emerging spots in the active regions instead of connecting with something far away is channeled very close to the surface or to a nearby spot. The force that contains this new field is probably the existing magnetic field of the old spots. There is a whole class of sunspots either without penumbras altogether or with sharply sheared penumbras which characteristically produce big flares, and I think that they are characterized by this type of field that is sharply turned over and is completely force free and completely different from what it would normally be. 